\title{
Friebel, Harry
}

\section{Geschlecht, Elternschaft und Weiterbildungsteilnahme}

Erziehungswissenschaft 25 (2014) 48, S. 129-142

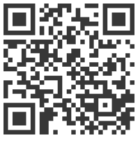

Quellenangabe/ Reference:

Friebel, Harry: Geschlecht, Elternschaft und Weiterbildungsteilnahme - In: Erziehungswissenschaft 25 (2014) 48, S. 129-142 - URN: urn:nbn:de:0111-opus-95630 - DOI: 10.25656/01:9563

https://nbn-resolving.org/urn:nbn:de:0111-opus-95630

https://doi.org/10.25656/01:9563

in Kooperation mit / in cooperation with:

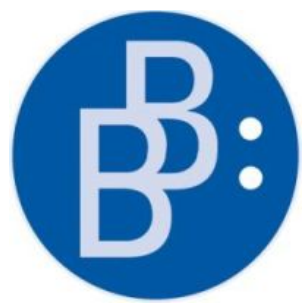

https://www.budrich.de

\section{Nutzungsbedingungen}

Gewährt wird ein nicht exklusives, nicht übertragbares, persönliches und beschränktes Recht auf Nutzung dieses Dokuments. Dieses Dokument ist ausschließlich für den persönlichen, nicht-kommerziellen Gebrauch bestimmt. Die Nutzung stellt keine Übertragung des Eigentumsrechts an diesem Dokument dar und gilt vorbehaltlich der folgenden Einschränkungen: Auf sämtlichen Kopien dieses Dokuments müssen alle Urheberrechtshinweise und sonstigen Hinweise auf gesetzlichen Schutz beibehalten werden. Sie dürfen dieses Dokument nicht in irgendeiner Weise abändern, noch dürfen Sie dieses Dokument für öffentliche oder kommerzielle Zwecke vervielfältigen, öffentlich ausstellen, aufführen, vertreiben oder anderweitig nutzen.

Mit der Verwendung dieses Dokuments erkennen Sie die Nutzungsbedingungen an.

\section{Terms of use}

We grant a non-exclusive, non-transferable, individual and limited right to using this document.

This document is solely intended for your personal, non-commercial use. Use of this document does not include any transfer of property rights and it is conditional to the following limitations: All of the copies of this documents must retain all copyright information and other information regarding legal protection. You are not allowed to alter this document in any way, to copy it for public or commercial purposes, to exhibit the document in public, to perform, distribute or otherwise use the document in public.

By using this particular document, you accept the above-stated conditions of use.

\section{Kontakt / Contact:}

\section{peDOCS}

DIPF | Leibniz-Institut für Bildungsforschung und Bildungsinformation Informationszentrum (IZ) Bildung

E-Mail: pedocs@dipf.de

Internet: www.pedocs.de

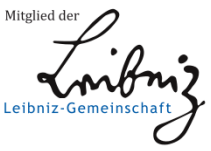




\section{Erziehungswissenschaft}

Mitteilungen der Deutschen Gesellschaft

für Erziehungswissenschaft (DGfE)

Heft 48

25. Jahrgang 2014

ISSN 0938-5363

Verlag Barbara Budrich 


\section{INHALTSVERZEICHNIS}

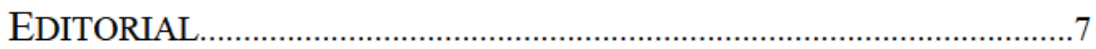

BEITRÄGE ZUM 50. JUBILÄUM DER DGFE

Britta Behm

50 Jahre „Deutsche Gesellschaft für Erziehungswissenschaft“ (DGfE).

Gedanken zu Jubiläum und Forschungslücken .

LAUDATIONES

Ingrid Miethe

Laudatio für Prof. Dr. Hannelore Faulstich-Wieland anlässlich der

Verleihung des Ernst-Christian-Trapp-Preis.

Hannelore Faulstich-Wieland

Dankesrede für den Ernst-Christian-Trapp-Preis

Dietrich Benner, Tina Hascher \& Werner Thole

Laudatio für Prof. Dr. Eckhard Klieme anlässlich der Verleihung des

DGfE Forschungspreises 2014.

Eckhard Klieme

Dankesrede zur Verleihung des Forschungspreises der DGfE.

Stefan Aufenanger

Laudatio für Martin Spiewak anlässlich der Verleihung des

Medienpreis der DGfE.

Isabell van Ackeren, Carola Groppe \& Ivo Züchner

Laudatio für Katrin Kaufmann und Sarah Widany anlässlich der

Verleihung des Förderpreises der DGfE

Ingrid Kunze \& Norbert Ricken

Laudatio für Ruprecht Mattig anlässlich der Verleihung des

Förderpreises der DGfE.

Marcelo Caruso

Laudatio für Prof. i.R. Dr. Marianne Krüger-Potratz anlässlich der

Verleihung der Ehrenmitgliedschaft der DGfE.

Marcelo Caruso

Laudatio für Prof. Dr. Dieter Lenzen anlässlich der Verleihung der

Ehrenmitgliedschaft der DGfE. 
Autorinnen und Autoren der Laudationes

BEITRÄGE ÜBER DIE NACHWUCHSFÖRDERUNG IN DEN

SEKTIONEN

Nina Blasse \& Doris Wittek

Die Situation des wissenschaftlichen ,Nachwuchses“ im Fach

Erziehungswissenschaft -2014 revisited.

Petra Götte \& Jörg-W.

Nachwuchsförderung in der Sektion 1 - Historische Bildungsforschung.....73

Selma Haupt \& Rita Molzberger

Forum Erziehungsphilosophie 75

Imke von Bargen, Christian Brüggemann, Corinne Ruesch \&

Anne Schmuki

Nachwuchsförderung in der Sektion 3 - Interkulturelle und

International Vergleichende Erziehungswissenschaft (SIIVE).

Anna-Katharina Praetorius

Nachwuchsförderung in der Sektion 4 - Empirische Bildungsforschung.....80 80

Erika Gericke, Martina Hinze, Silke Kassebaum \& Alexander Schnarr

Die hierarchiefreie Forschungsgemeinschaft als

Professionalisierungsinstanz in der Wissenschaft.

Oktay Bilgi \& Gloria von Papen Robredo

Erfolgreiche Dissertation durch strukturierte Promotion?

Sarah Henn \& Kathrin Schulze

Das Netzwerk ,Junge Wissenschaft der Sozialen Arbeit“ als Förderung

der , eigenen' Stimme.

Michael Lichtblau

Nachwuchsarbeit in der Kommission Pädagogik der frühen Kindheit 88

Cornelia Maier-Gutheil, Marion Fleige, Sylvana Dietel \&

Bettina Thöne-Geyer

Werkstatt Forschungsmethoden

Mandy Schiefner-Rohs, Sandra Aßmann \& Sandra Hofhues

Nachwuchs früh beteiligen: Formen der Nachwuchsförderung in der

Sektion 12 - Medienpädagogik. 


\section{BEITRÄGE}

Klaus Zierer, Hubert Ertl, David Phillips \& Rudolf Tippelt

Profillinien erziehungswissenschaftlicher Zeitschriften. Eine

Orientierungshilfe

Harry Friebel

Geschlecht, Elternschaft und Weiterbildungsteilnahme

MITTEILUNGEN DES VORSTANDS

Rechenschaftsbericht

Beschlussvorlage.

DGfE-Summer School 2014

\section{BERICHTE AUS DEN SEKTIONEN}

Sektion 2 -Allgemeine Erziehungswissenschaft.

Sektion 3 - International und Interkulturell Vergleichende

Erziehungswissenschaft (SIIVE)

Sektion 4-Empirische Bildungsforschung.

Sektion 8 - Sozialpädagogik und Pädagogik der frühen Kindheit.

Sektion 11 - Frauen- und Geschlechterforschung in der

Erziehungswissenschaft .... 174

Sektion 12 - Medienpädagogik. 176

NOTIZEN

Aus der Forschung. 177

Aus Wissenschaft und Lehre 182

TAGUNGSKALENDER 183

\section{PERSONALIA}

Nachruf auf Prof. Dr. Gabriele Faust......

Nachruf auf Prof. Dr. Jens Naumann 


\title{
Geschlecht, Elternschaft und Weiterbildungsteilnahme
}

\author{
Harry Friebel
}

Weiterbildungsteilnahme in den Kategorien Geschlecht und Elternschaft zu diskutieren heißt, lebens- und arbeitsweltliche Kontexte zu reflektieren. Inklusion in die und Exklusion aus der Weiterbildung sind nur zu verstehen, wenn wir biographische „Regulative“ (Wittpoth 2009: 779) der Teilnahme und Nicht-Teilnahme berücksichtigen. Insofern ist jede Teilnahmediskussion zugleich Kontextdiskussion. Wenn zum Beispiel im jüngsten AES-Monitoring zur Weiterbildung in Deutschland zu lesen ist, dass 39\% der befragten Männer an betrieblicher, $18 \%$ an nicht-betrieblicher, aber nur $31 \%$ der befragten Frauen an betrieblicher, doch $25 \%$ an nicht-betrieblicher Weiterbildung teilgenommen haben (Leven et al. 2013: 80) dann sind diese Unterschiede der Teilnahmequoten nicht selbsterklärend - sie sind Ausdruck geschlechts- und biographiespezifischer Lebenszusammenhänge.

Die Frage dieses Artikels, ob Chancengleichheit der Geschlechter in der Weiterbildungsteilnahme erreicht wurde, wird hier deshalb in der Tradition der Lebenslaufforschung diskutiert: Es geht um Optionen und Restriktionen der individuellen Weiterbildungsbeteiligung im Kontext von Familien- und Erwerbsarbeit. Dabei berufen wir uns auf den Ursprung der Life-Course-Theory in Elders berühmter US-Studie „Children of the Great Depression“ (vgl. Elder 1974). Elder entwickelte eine Theorie mit grundlegenden Prinzipien zur Einheit von Individualisierung und Institutionalisierung (Friebel 2008b: 481) innerhalb der gesamten Lebensspanne, d.h. zur Gleichzeitigkeit der Logik des Subjekts (Individuen gestalten ihr eigenes Leben auf der Grundlage ihrer Entscheidungen und Handlungen innerhalb ihrer Möglichkeiten) und der Logik der Struktur (der Lebenslauf von Individuen ist sowohl eingebettet als auch berührt durch die historische Zeit und ihre Ereignisse).

Die Diskussion des Zusammenhangs von Geschlecht, Elternschaft und Weiterbildungsteilnahme generieren wir in zwei Schritten: Zuerst erörtern wir den Zusammenhang im Kontext der Familiengründung als Transitionsprozess. Danach fokussieren wir Aspekte der geschlechtsspezifischen Beteiligungsregulation in und durch die Erwerbsarbeit im Rahmen einer Typologie.

\section{Empirische Datengrundlage}

Wir diskutieren Befunde der Längsschnittstudie „Hamburger Biografie- und Lebenslaufpanel"(HBLP) - ein Sample der Hamburger Schulabschlusskorte von 1979 (vgl. Friebel 2000; Friebel 2008a). In bisher 21 Erhebungswellen mit einem regelmäßigen Zeitabstand von etwa eineinhalb Jahren - begleite- 
ten wir diese Untersuchungsgruppe von 1980 bis 2012 auf der Basis von Fragebogenbefragungen (quantitativ) und mit problemzentrierten Interviews (qualitativ) prospektiv forschend, um Veränderungen in der Bildungs- und Weiterbildungsbeteiligung auf lange Sicht erfassen zu können. 130 Personen, die 1979 das allgemeinbildende Schulwesen in Haupt- und Realschulen sowie Gymnasien in Hamburg abgeschlossen hatten, nahmen bis einschließlich der 21. Erhebungswelle (2012) an der Untersuchung teil. Der ursprüngliche Stichprobenumfang lag in der ersten Welle (1980) bei 252 Personen. ${ }^{1}$ In der sechsten Welle (1988) wurde die Stichprobe um eine bereits 1979 von uns vorab gezogene Ergänzungsstichprobe von 123 Personen erweitert, um die „Panel-Sterblichkeit“ (vgl. Haunberger 2011) in unserer Längsschnittstudie teilweise zu kompensieren (vgl. DIW 1998). Die Gesamtheit des zur Diskussion stehenden Samples nennen wir Gesamtsample. Von ihnen liegen Verlaufsdaten zu zentralen biographischen Erfahrungen von 1980 bis 2012 vor. Zusätzlich wurden pro Welle mit einem Subsample von jeweils ca. 25 Angehörigen des Gesamtsamples problemzentrierte Interviews durchgeführt: Wir nennen dieses Subsample Intensivsample. Die Auswahl des Intensivsamples richtete sich nach problembezogenen und theoretischen Überlegungen (vgl. Glaser/Strauss 1967; Strauss/Corbin 1990).

Die Sampleangehörigen sind aktuell etwa 50 Jahre alt; verheiratet sind ca. zwei Drittel, Eltern sind ca. sieben Zehntel.

\section{Weiterbildungsteilnahme im Kontext von Familie und Erwerbsarbeit}

Wir wählen den Begriff „Kontext“, um ausgewählte lebensweltliche hemmende und fördernde Bedingungen bzw. Regulative des Weiterbildungsverhaltens zu thematisieren - insbesondere hinsichtlich der Frage nach geschlechtstypischen Effekten der Statuspassagen Familienbildung und Erwerbsarbeit. Der Lebenslauf als Sequenz von Statusfigurationen impliziert Chancen und Risiken, Wendepunkte und Passagen, die sich in unterschiedlicher Weise auf das Weiterbildungsverhalten auswirken können. Unsere These von der Kontextabhängigkeit der Weiterbildungsteilnahme stellt nicht in Frage, dass Lernen, Bildung und Weiterbildung biographische Eigenleistungen sind - sondern soll nur die biographische und lebensweltliche ,Rahmung' dieser Eigenleistungen aufzeigen. Werden diese Kontextparameter

Die Stichprobe ist eine zufallsgenerierte Klumpenauswahl: In drei sozialstrukturell unterschiedlich zusammengesetzten Stadtteilen Hamburgs wurden jeweils drei Schulen verschiedener Schulformen ausgewählt. Mittels Zufallszahlen wurde das Sample aus den Klassenlisten der Schulabsolventeninnen und Schulabsolventen generiert. 
nicht beachtet, so besteht das Risiko einer Individualisierung der (Nicht-) Teilnahme.

„Jeder sollte bereit sein, sich ständig weiterzubilden“ sagten 91\% (2000) bzw. 94\% (2005) aller Befragten im repräsentativen Berichtssystem Weiterbildung (BSW) (vgl. BMBF 2003: 79; BMBF 2006: 258) auf eine Frage nach der Bedeutung der Weiterbildung. Die tatsächliche Weiterbildungsteilnahmequote in Deutschland lag aber regelmäßig unterhalb von 50\% (BMBF 2013: 7; Bilger et al. 2013: 29; Rosenbladt/Bilger 2011: 27). Was behindert, was fördert die Weiterbildungsteilnahme im Lebenszusammenhang? Wie ist die Realisierungslücke zu verstehen? Für eine Beantwortung dieser Fragen sind erst empirisch gestützte Reflexionen über Kontexteffekte in der Lebenslaufperspektive zielführend!

\section{$2.1 \quad$ Weiterbildungsteilnahme und Familiengründung}

Die Kurvendarstellung ist ein stilisiertes Bild zur Teilnahme des Untersuchungssamples an organisierter Weiterbildung in Form von Lehrgängen, Kursen und sonstigen Schulungen etc. - also an „,non-formaler Bildung“ - im Lebenszusammenhang. ${ }^{2}$

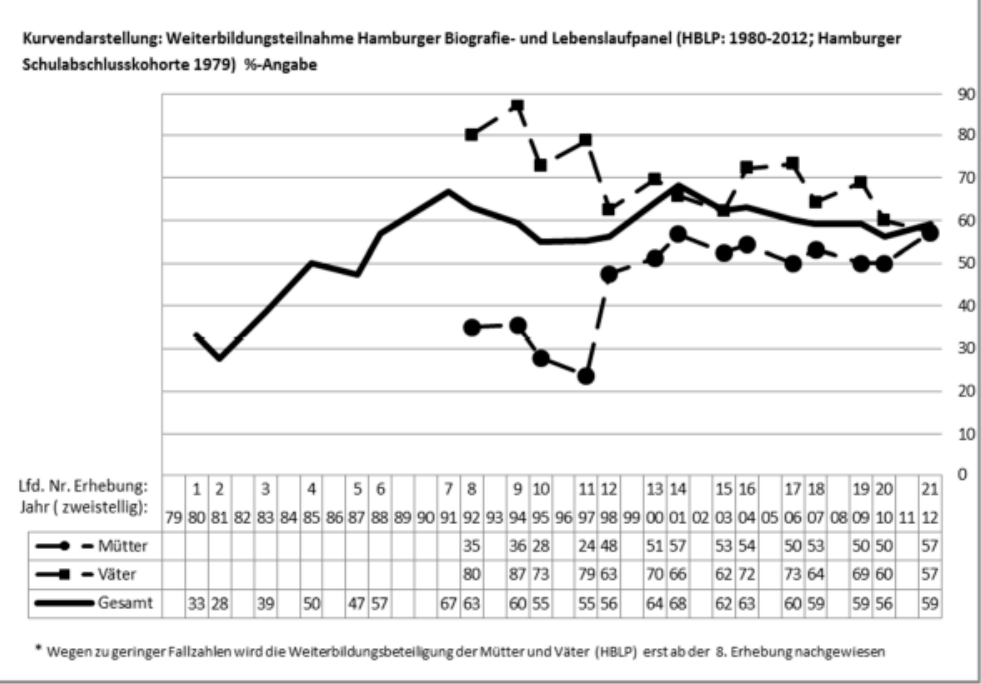

Quelle: eigene Darstellung

2 Eine Differenzierung zwischen beruflicher und nicht beruflicher Weiterbildungsteilnahme wird nicht vorgenommen. 
Die Teilnahmequoten des Samples in den 21 Wellen werden in Kurven (und in einem Tabellenteil darunter) dargestellt. ${ }^{3}$ Die Weiterbildungsteilnahme der Befragten insgesamt (fette Kurve) des HBLP begann im Prozess der beruflichen Qualifizierung 1980 auf niedrigem Niveau und steigerte sich fast kontinuierlich bis 1991 (von 33\% = erste Welle bis 67\% = siebte Welle). Danach fiel das Teilnahmeniveau bis zur zehnten Welle fast stetig und verblieb in den Folgewellen auf diesem Niveau. Etwa ab der 13. Welle sehen wir wieder ansteigende Teilnahmefrequenzen - danach flachte die Teilnahmehäufigkeit erneut etwas $a b$.

Die intertemporale Reduktion der Weiterbildungsquoten verweist auf den biographischen Zusammenhang zwischen Elternschaft, Geschlecht und Weiterbildung im Lebenszusammenhang. Es ist ein "doing gender" (vgl. West/ Zimmermann 1987) - als soziale Konstruktion der Geschlechterverhältnisse im Lebenslauf (Krüger 2002: 63) - der Weiterbildungsteilnahme: Um diese soziale Konstruktion im Verhältnis der Geschlechter im Lebenszusammenhang anschaulich dokumentieren zu können, haben wir die Weiterbildungsverlaufsmuster in Abhängigkeit vom Geschlecht der Elternschaft konstruiert. Diese Prozessgrammatik spiegelt sich in den beiden ergänzenden Kurven wieder: Die Kurve der Männer, die im Laufe des Untersuchungszeitraums Väter geworden sind, ist ab der achten Erhebungswelle hinsichtlich der Weiterbildungsteilnahme signifikant überdurchschnittlich. Sie dokumentiert bis zur 20. Welle nahezu stetig überdurchschnittliche Teilnahmequoten. Die Kurve der Frauen, die im Laufe des Untersuchungszeitraums Mütter geworden sind, ist hinsichtlich der Weiterbildungsteilnahme ab der 8. Erhebungswelle signifikant unterdurchschnittlich. Ab der 14. Welle erreicht sie wieder ein etwa durchschnittliches Niveau. Erst zur 21. Welle liegen die Mütter und die Väter auf einem ähnlichen Teilnahmeniveau. Die Kurvendarstellung dokumentiert eine signifikante Scherenöffnung zwischen einer überdurchschnittlichen Weiterbildungsaktivität der Väter und einer Weiterbildungsabstinenz der Mütter. Zwischen der achten und der 21. Welle liegen ca. 20 Jahre Lebenslauf, Biographie, Karriere voller Aktivität oder Abstinenz. Die Kurvendarstellung zeigt - in prozessualer Perspektive - wie Mütter gewissermaßen gefangen sind in einer Verlaufskurve, die Krüger als ,prozessuale Diskriminierung“ (Krüger 1995: 133) beschreibt. Das Geschlecht lagert sich als Strukturkategorie sowohl in die beruflichen Qualifizierungsprozesse als auch in die Familienbildungsprozesse in typischer Weise ein. Das Geschlecht „Frau" bedeutet beim Übergang in den Beruf, Vorabzüge" in der Verwertung von Bildungsabschlüssen schon vor der Familiengründung. Und das Ge-

Wir haben drei Kurven in die Darstellung intergriert: Die durchgehende, fette Kurve symbolisiert die durchschnittliche Weiterbildungsquoten des Gesamtsamples pro Erhebungswelle. Die beiden zusätzlichen Kurven berichten über die Weiterbildungsteilnahmequoten von Frauen und Männer des Samples, nachdem sie Mütter und Väter wurden. 
schlecht „Frau“ erfährt mit dem biographischen Einstieg in die Elternschaft eine „Soll-Bruchstelle“ (Friebel 2007: 42) in der Erwerbsarbeit und der Weiterbildung: Diese Diskriminierung kommt „dann zusätzlich hinzu“ (ebd.). Krüger fordert deshalb - bezogen auf die Lebensläufe gleichermaßen für Frau und Mann - die wechselseitigen Kontexteffekte von Arbeitswelt und Familie zu sehen:

„Erst die Verknüpfungsprinzipien beider Institutionen machen die Gegenläufigkeit der Ungleichheitsspirale sichtbar: Das positive Aufaddieren von Geschlecht und Familie im männlichen gegenüber der umgekehrt proportionalen Negativentwicklung im weiblichen Lebensverlauf." (Krüger 1995: 144, Hervorhebung im Original)

Es ist in der Kurvendarstellung empirisch wahrscheinlich, dass sich die Differenzwerte in der ,Berg- und Talfahrt' der Väter- und Mütter-Kurven in Abhängigkeit von den Bildungs- und Qualifikationsprofilen dieser beiden Subgruppen verstärken oder vermindern können. In unserem Sample gibt es eine leichte geschlechtlich gebundene Qualifikationsverteilung. Die Frauen und Männer des Samples haben zwar jeweils ausgesprochen ähnliche Bildungsressourcen in ihren Herkunftsfamilien erfahren, aber hinsichtlich der Schulbildungsabschlüsse und der Berufsqualifikationen existieren gewisse Unterschiede: Die Männer im Sample sind bezogen auf den Schulabschluss Abitur und das Hochschulstudium etwas überrepräsentiert - die Frauen hingegen beim Schulabschluss Hauptschule und der nichtakademischen Berufsausbildung. Weil nun alle(!) empirischen Studien berichten, dass mehr Bildung mehr Weiterbildung generiert - und umgekehrt - würde eine Gleichverteilung der Bildung zwischen den Männern und Frauen im Sample die ,Berg und Talfahrt' der Kurven weniger dramatisch ausfallen lassen. Diese Kurvendarstellung ist ohnehin nur eine Aggregation vielfältiger lebensweltlich unterschiedlicher Einzelfälle im Prozess - ein aggregierendes Schema mit heuristischem Wert.

Im Rahmen unserer parallel zu den standardisierten Fragebogenbefragungen durchgeführten problemzentrierten Interviews mit dem Intensivsample gaben die Mütter und die Väter prototypische ,Spielregeln' ihrer jeweiligen Familiensituation in der Phase der Familiengründung zu Protokoll:

- Mutter: „Als das erste Kind kam, da hab ich alle Weiterbildung erst mal aufs Eis gelegt ... für die Familie“.

- Vater: „Als das erste Kind kam, da hab ich erst mal alles an Weiterbildung gemacht ... für die Familie“.

Vereinfacht ausgedrückt, wirkt das soziale Geschlecht im Kontext von Elternschaft als Strukturgeber der Weiterbildungsteilnahme.

Die geschlechtsspezifischen Verteilungen insgesamt zur Weiterbildungsteilnahme der Sampleangehörigen erscheinen hingegen ausgesprochen unauffällig. Das liegt eben daran, 
- dass die im Untersuchungsverlauf Mütter gewordenen Frauen erheblich unterdurchschnittliche Teilnahmequoten - wie in der Kurvendarstellung dokumentiert - hatten, dagegen die Frauen ohne Kinder signifikant überdurchschnittlich an Weiterbildung teilgenommen haben,

- dass die im Untersuchungszeitraum Väter gewordenen Männer erheblich überdurchschnittliche Teilnahmequoten hatten - wie in der Kurvendarstellung dokumentiert -, aber die Männer ohne Kinder signifikant unterdurchschnittlich an Weiterbildung teilgenommen haben.

Erst mit unserer prozessgestützten prospektiven Ermittlung von mikrostrukturellen Daten desselben Samples im Rahmen eines Panels lassen sich derartige biographiespezifische Ergebnisse gewinnen.

Auch im BSW/AES-Bericht über Weiterbildungsteilnahme 2010 wird der Zusammenhang von Erwerbsarbeit, Familie, Geschlecht und Weiterbildung aufgegriffen: „Familiäre Verpflichtungen sind mit einem Anteil von $25 \%$ die von Frauen am häufigsten genannte Weiterbildungsbarriere (Männer 5\%)“. Zudem führen die Autorinnen und Autoren aus, dass bei den Erwerbstätigen dieser Hemmfaktor bestehen bleibt:

„So nennen $41 \%$ der erwerbstätigen Frauen mit Kindern unter 14 Jahren im Haushalt familiäre Verpflichtungen als wichtigste Weiterbildungsbarriere, während es von den erwachsenen Männern dieser Teilgruppe lediglich 11\% sind." (Rosenbladt/Bilger 2011: 168)

Und im AES-Bericht über Weiterbildungsteilnahme 2012 ist gleichlautend zu lesen: „Frauen sehen familiäre Verpflichtungen etwa viermal so oft wie Männer als wichtigste Weiterbildungsbarriere an (16\% vs. $4 \%$ )" (Bilger et.al. 2013: 220). Die prozessgestützten Ergebnisse der Kurvenanalyse unserer HBLP-Studie und die bivariaten sowie multivariaten zeitpunktbezogenen Befunde der BSW/AES-Studie können verfugt werden zu einem Gesamtbild der Reproduktion typisch weiblicher und männlicher Lebensläufe in der „Moderne" (vgl. Agentur 2011). Strukturell ähnliche Befunde der empirischen Weiterbildungsstudie des Bundesinstituts für Berufsbildung von 2002 zum Doing Gender veranlassen Stürzer im Gender-Datenreport zu dem Hinweis, dass für die häufig diskontinuierliche Erwerbsbiographie von Müttern eine ,vermehrte Partizipation an beruflicher Weiterbildung zur Kompensation fehlender Qualifizierungsmöglichkeiten on the job wünschenswert [sei], um ihre Chancengleichheit zu verbessern" (Stürzer 2005: 85). Und die Autoreninnen und Autoren der Sachverständigenkommission des ersten (Gender-)Gleichstellungsberichts der Bundesrepublik verweisen in diesem Kontext auf die Lebenslaufperspektive:

„Eine Ehescheidung, der Wechsel in Teilzeit, eine Erwerbsunterbrechung oder eine unterlassene Weiterbildung können ,Narben' hinterlassen (,scar effects'), die lange nachwirken." (BMFSFJ 2011: 45) 
Sie appellieren an die Politik, dass nur eine aktive Lebenslauf- und Gleichstellungspolitik Rahmenbedingungen für eine Überwindung dieses Doing Gender bereitstellen kann.

\subsection{Weiterbildungsteilnahme und Arbeitswelt}

Der Arbeitsplatz als Lernwelt: In den problemzentrierten Interviews fragten wir: „Was fördert Sie, Weiterbildung zu machen?" Typische Antworten der Sampleangehörigen mit Arbeitgebersupport waren:

- „Das was hier meine Firma macht ... und Förderung der Mitarbeiter ähm Gebühren übernehmen, hier interne Fortbildungen. Also das heißt: ich kann mich jederzeit für ... wir haben ne ziemlich komplexe, interne Fortbildung ... also da ist meine Firma echt schon sehr positiv aufgestellt.“

- „Wir kriegen jedes Jahr zum Anfang des Jahres, kriegen wir nen Fortbildungskatalog in jede Einheit auf den Stationen. Und da können wir alle reingucken und können für uns äh entscheiden was wir als Fortbildung nehmen wollen. Äh da gibt es dann in Neu ... aber dann gibt es auch Erweiterungsbausteine und da guck ich dann immer wo ich ähm mich sehe."

- „Also mich, es fördert schon irgendwie, wenn der Arbeitgeber ne Fortbildung mir finanziert und auch in der Arbeitszeit stattfindet lässt ... Also, das ganz klar, ich hab ja die letzten Fortbildungen gemacht, waren alle im Rahmen meiner Arbeitszeit...und vom Arbeitgeber auch finanziert. Also, also die kamen sogar ins Haus [Inhouse-Schulung, H.F.] bei uns. So. Also, ich wurde mit anderen Kollegen zusammen geschult. Das war, das auf jeden Fall förderlich ...dann nehm ich dran teil.“

- „Na logischerweise Kostenbeteiligung des Arbeitergebers, Freistellung, solche Geschichten ... Entwicklungsmöglichkeiten, Karriere, Aufstieg, finde ich alles motivierend für Weiterbildung ...Da bin ich richtig gut bestückt von meinem Arbeitgeber."

Die betriebliche Weiterbildung ist laut jüngster Befunde der AES-Studie „der Teilbereich der Weiterbildung mit der höchsten Reichweite: mit einem Anteil von 69\% an allen Weiterbildungsaktivitäten" (Behringer et al. 2013: 139). In unserer HBLP-Studie liegt - bezogen auf den Zeitraum von der 18. bis zur 21. Welle - der Anteil der weiterbildungsaktiven Sampleangehörigen, die an betrieblicher Weiterbildung teilgenommen haben, zwischen 53\% (18.W.) und $61 \%$ (21.Welle). ${ }^{4}$ ge jeweils identischer Fragenformulierungen im Fragebogen ermittelt werden konnten. 
Wir greifen für die Analyse des Arbeitgeber-Supports als Kontext in exemplarischer Absicht auf die oben beschriebenen von Elder aufgezeigten grundlegenden Prinzipien zur Einheit von Individualisierung und Institutionalisierung im Lebenslauf zurück und konstruieren eine Typologie der Weiterbildungsbeteiligung, die eine ,individuenzentrierte“ (Hoffmann 2012: 155) Sicht überwinden helfen kann: Handelndes Individuum und struktureller Kontext stehen in einem Verhältnis - Individualaussagen und Kontextaussagen müssen zusammengefügt werden. ${ }^{5}$ Deshalb zielen wir auf diese typologische Analyse des Zusammenhangs von Individuum und Struktur. Einen ersten Schritt in dieser Richtung stellen wir hier vor. Mit den Ergebnissen der Typologie ermitteln wir gewissermaßen eine Verfugung zwischen Idealtypen als ,einheitliche Gedankengebilde“ (Weber 1904/1988: 191) und Realtypen als Ausprägungen alltäglicher Wirklichkeit.

Um die Komplexität dieser Analyse zunächst noch gering zu halten, führen wir - am Beispiel des exemplarisch ausgewählten Referenzzeitraums von der 18. bis zur 21. Welle - nur drei weitere Kontextvariablen ein:

- Arbeitsvertrag der Sampleangehörigen: Etwa jeweils die Hälfte der Befragten befand sich in einem Vollzeitarbeitsverhältnis - ein Fünftel in einem Teilzeitarbeitsverhältnis. Ein Zehntel war arbeitslos und ebenfalls jeweils ungefähr ein Zehntel war selbstständig erwerbstätig oder nichterwerbstätig. Zusammen genommen waren etwa zwei Drittel der Befragten abhängig erwerbstätig in einem Vollzeitarbeitsverhältnis - oder selbstständig erwerbstätig. ${ }^{\text {. }}$

- Weiterbildungsangebot des Arbeitgebers: Etwa jeweils die Hälfte der Befragten meinte in allen vier Wellen, dass das zur Verfügung gestellte Weiterbildungsangebot voll ausreichend war. Jeweils ungefähr ein Viertel sagte, dass das vorhandene Weiterbildungsangebot nicht ausreichend war oder gar kein Angebot existiere. Etwa insgesamt drei Viertel der Befragten erfuhren also ein mehr oder weniger ausreichendes Weiterbildungsangebot seitens der Arbeitgeber.

- Finanzierung der Weiterbildung durch Arbeitgeber: Ca. ein Drittel der Befragten teilte mit, vom Arbeitgeber die Weiterbildungsteilnahme mehrmals finanziert bekommen zu haben - ein Fünftel einmal. Ungefähr zwei Fünftel stellten fest, keine Finanzierung durch den Arbeitgeber erhalten zu haben. Etwas mehr als die Hälfte der Befragten erfuhr also eine mehr oder weniger stetige Arbeitgeberfinanzierung für ihre Weiterbildungsteilnahme.

5 Diese Diskussion wird insbesondere im angelsächsischen Sprachraum auch bezogen auf die Frage nach dem Verhältnis zwischen „agency and structure“ (Anisef et al. 2000, S. 20) geführt.

6 Vereinfachend unterstellen wir - auch auf der Grundlage ergänzender Daten - für diese Gruppe einen Vollerwerbsarbeitsstatus. 
Die Verteilungen der einzelnen Ausprägungen aller drei Variablen sind in den vier hier berücksichtigten Erhebungswellen ausgesprochen stabil.

Im nächsten Schritt kombinierten wir die Kontextparameter der Weiterbildungsteilnahme mit den personenbezogenen Variablen Geschlecht, Schulabschluss, Berufsstatus, Elternschaft zu einer regelgeleiteten Typologie. Unsere Typenbildung (vgl. Kluge/Kelle 2010; Gerhardt 1995) ist fall- und prozessorientiert - sie ist fallkontrastierend angelegt (vgl. Kluge 1999). Wir konstruieren auf der Grundlage der Ausprägungen der drei Kontextvariablen drei maximale Gegensatzpaare - analog eines semantischen Differentials. In der Typologie wurden entsprechende maximale Gegensatzpaare definiert ${ }^{7}$ :

- $\quad$ hinsichtlich des Arbeitsvertrages = Vollzeit vs. Teilzeit

- hinsichtlich des Weiterbildungsangebots vom Arbeitgeber $=\mathrm{ja}$, ausreichend vs. nein, kein

- hinsichtlich der Weiterbildungsfinanzierung durch Arbeitgeber $=\mathrm{ja}$, mehrmals vs. nein, keinmal.

Die empirische Kovariation der biographie- bzw. personenbezogenen Variablen und der drei Kontextvariablen verweist auf zwei kontrastierende Typen:

- Erwerbstätige Väter mit dem 1979 erworbenen Realschulabschluss oder dem Abitur, die im ersten oder zweiten Bildungsweg einen Hochschulabschluss erworben haben, sind kontinuierlich überrepräsentiert im Vollzeit-Arbeitsverhältnis, berichten kontinuierlich über ein ausreichendes Weiterbildungsangebot des Arbeitgebers und über eine mehrmalige Weiterbildungsfinanzierung durch den Arbeitgeber: Sie haben regelmäßig von der 18. bis 21 . Welle an Weiterbildungsveranstaltungen teilgenommen.

- Erwerbstätige Mütter, die 1979 keinen weiterführenden Schulabschluss erwarben, die nur eine grundständige Berufsausbildung - betrieblich oder vollzeitschulisch - abschlossen, sind kontinuierlich überrepräsentiert in einem Teilzeit-Arbeitsverhältnis, berichten kontinuierlich, dass der Arbeitgeber weder ein Weiterbildungsangebot vorhält noch die Finanzierung von Weiterbildungsteilnahmen übernimmt: Nur für den Fall des Teilzeit-Arbeitsvertrages in der 21. Welle gibt es Struktur- und Verlaufsinkonsistenzen in der Typenbildung: Diese Mütter berichten über eine gelegentliche Teilnahme an Weiterbildungsveranstaltungen - ansonsten haben sie von der 18. bis 21. Welle keine Weiterbildungsteilnahme.

Die Ergebnisse der Typologie verweisen auf eine soziale Spaltung in einer wechselseitigen Durchdringung von Biographie, Geschlecht und Gelegen-

Wegen des kleinen Samples wurde die Konstruktion der maximalen Gegensätze mit Rücksicht auf die Anzahl der Ausprägungen gelegt. 
heitsstruktur. Die Lern- und Lebenswelt der Erwerbsarbeit prozessiert Inklusion in die und Exklusion aus der Weiterbildungsteilnahme im Kontext der drei eingeführten Kontextvariablen. Das Doing Gender der Erwerbsarbeitswelt wird also in der Weiterbildungsteilnahme fortgeführt. Die Statuspassage Familiengründung hat für Mütter und Väter - wie bereits in der Kurvendarstellung dokumentiert - unterschiedliche Anschlussereignisse: Das aktive (Teilnahme) und das passive (Nicht-Teilnahme)Weiterbildungshandeln einerseits und die Kontextbedingungen der Weiterbildungsgelegenheiten andererseits treffen sich im Lebenszusammenhang unmittelbar. Wir konstruierten in der Typologie zwei „Verlaufsmuster" ${ }^{\text {" }}$ in einer methodisch noch vereinfachenden Weise, aber bereits zielführend: Obwohl beide Typen in ihrer Abhängigkeit von heteronomen Aktivitätsbedingungen ${ }^{9}$ (Arbeitgebersupport) bedingt sind, erscheint der eine Verlauf als ,positive Verlaufskurve“ (Teilnahme), der andere als ,negative Verlaufskurve“ (Nicht-Teilnahme). In Verknüpfung mit den obigen Prozessbefunden zum Doing Gender der Weiterbildungsteilnahme erkennen wir, dass Handlungsschemata, Geschlecht und Bildungsbiographie mit der jeweiligen Lebenswelt und den Institutionenkontexten so zusammenführt werden müssen, dass sowohl voluntaristische Subjektkonzepte als auch deterministische Strukturkonzepte überwunden werden können.

Eine mögliche Bedeutung für einen Wandel der Weiterbildungsteilnahme im Kontext modernisierter Arbeits- und Lebensverläufe signalisieren allerdings Befunde des BSW/AES-Weiterbildungsmonitoring hinsichtlich der Weiterbildungsteilnahme teilzeitbeschäftigter Frauen:

„Während die Weiterbildungsbeteiligung der Vollzeit-Beschäftigten - Männer wie Frauen - zurückgegangen ist, hat die Weiterbildungsbeteiligung der teilzeitbeschäftigten Frauen sogar zugenommen." (Bilger/Rosenbladt 2011: 62)

Keller et al. bezeichnen die ,reguläre“ Teilzeit der Frauen als „Neues Normalarbeitsverhältnis"(Keller et al. 2012: 17) der Frauen. Es ist deshalb zu fragen, ob dieser angenommene Struktur- und Bedeutungswandel atypischer Beschäftigung zu einer stärkeren Inklusion der betroffenen Frauen bzw. Mütter in den Bereich der betrieblichen Weiterbildung führt. Möglich ist dabei,

Schütze hat Anfang der 1980er Jahre in seinem Konzept „Prozessstrukturen des Lebenslaufs" Modelle des (passiven) „Erleidens" mit dem Begriff, ,negative Verlaufskurve " und des (aktiven) ,Handelns ${ }^{c c}$ der Biographieträgerinnen und Biographieträger vorgestellt (vgl. Schütze 1984).

9 Dabei „widerspricht" die subjektive Wahrnehmung (Logik des Subjekts) der Sampleangehörigen den faktischen Handlungsbedingungen (Logik der Struktur) (Helfferich 2012, S. 16). Die große Mehrheit der Weiterbildungsteilnehmenden stellt fest, dass sie sich selbst für ihre Teilnahme entschieden haben: Im Verhältnis von etwa 2:1 identifizieren sie sich (bezogen auf die 18. bis 21. Welle) überwiegend als Selbst-Aktivierende (ca. 50\%), nur sekundär als Arbeitgeber-Aktivierte (ca. $25 \%$ ). 
dass auch der in der obigen Kurvendarstellung dokumentierte Anstieg der Weiterbildungsbeteiligung der Mütter in unseren Sample auf das Niveau der Väter im Sample in der 21. Welle, diese Inklusionschancen aufzeigt.

\section{$3 \quad$ Fazit}

Die Lernentwicklung der Individuen selbst und der soziale Lernraum als institutionalisierter Rahmen müssen zusammen analysiert werden. Weiterbildung wird als Weiter-Bildung typischerweise in lebenslaufgebundenen Passagen (vgl. Mayer/Blossfeld 1990) generiert: Mütter sind deutlich unterrepräsentiert, Väter ebenso deutlich überrepräsentiert. Dieses Ungleichgewicht bei den Beteiligten ist doppelt problematisch: Zum einen steht es im Widerspruch zu dem politisch proklamierten Gleichstellungsanspruch, zum anderen führt es zu einer kumulativen Ausgrenzung von Müttern aus Chancenstrukturen einer befriedigenden Lebensführung und Erwerbsarbeit. Zudem muss angesichts der Tatsache, dass laut der AES-Studie 2012 69\% (vgl. Behringer et al. 2013) aller Weiterbildungsteilnahmefälle in Arbeitgeberhand stattgefunden haben, davon ausgegangen werden, dass der Angebotsbereich Weiterbildung überwiegend ein „closed-shop“ (Friebel 2010: 310) für Arbeitsplatzbesitzerinnen und Arbeitsplatzbesitzer ist.

Weiterbildungsteilnahme lässt sich erst in der Gleichzeitigkeit - und gegebenenfalls auch Widersprüchlichkeit - von Handlung und Gelegenheit nachvollziehen: Am Beispiel des Doing Gender (gerahmt durch das Doing Class dieser Teilnahme unserer Sampleangehörigen in ihrem Lebenslauf) und innerhalb ihrer sozialen Lebens- und Arbeitswelten haben wir eine Analyseperspektive für den prozessualen Zusammenhang von Selbst- und Fremdwirksamkeit aufgezeigt.

Mit der Fortführung dieser Längsschnittstudie zielen wir auf weitere Einsichten in das Verhältnis zwischen der Chance eines aktiven Gestaltens und den sozial konstruierten Regulativen der Weiterbildungsteilnahme.

Harry Friebel, Prof. Dr., ist Hochschullehrer für Soziologie an der Universität Hamburg.

\section{Literatur}

Agentur für Gleichstellung im ESF (Hrsg.) (2011): Gender-Aspekte in der betrieblichen Weiterbildung. Berlin: Agentur für Gleichstellung im ESF.

Anisef, Paul et al. (2008): Opportunity and Uncertainty. Life Course Experiences of the Class of ' 73 Toronto: University of Toronto. 
Behringer, Friederike/Bilger, Frauke/Schönfeld, Gudrun. (2013): Betriebliche Weiterbildung. In: Bilger, Frauke et al.(Hrsg.): Weiterbildungsverhalten in Deutschland. Resultate des Adult Education Survey 2012. Bielefeld: W. Bertelsmann Verlag, S. 139-163.

Bilger, Frauke/Gnahs, Dieter/Hartmann, Josef/Kuper, Harm (Hrsg.) (2013): Weiterbildungsverhalten in Deutschland. Resultate des Adult Education Survey, Bielefeld: W. Bertelsmann Verlag.

Bilger, Frauke/Rosenbladt, Bernhard von (2011): Aktuelle Entwicklung: Trends in verschiedenen Bevölkerungsgruppen. In: Bilger, F./Rosenbladt, B. von (Hrsg.): Weiterbildungsbeteiligung 2010. Trends und Analysen auf Basis des deutschen AES. Bielefeld: W. Bertelsmann Verlag, S. 61-69.

Bundesministerium für Bildung und Forschung (BMBF) (Hrsg.) (2003): Berichtssystem Weiterbildung VIII. Integrierter Gesamtbericht zur Weiterbildungssituation in Deutschland. Bonn: BMBF.

Bundesministerium für Bildung und Forschung (BMBF) (Hrsg.) (2006): Berichtssystem Weiterbildung IX. Integrierter Gesamtbericht zur Weiterbildungssituation in Deutschland. Bonn, Berlin: BMBF.

Bundesministerium für Bildung und Forschung (BMBF) (Hrsg.) (2013): Weiterbildungsverhalten in Deutschland. AES 2012 Trendbericht. Bonn: BMBF.

Bundesministerium für Familie, Senioren, Frauen und Jugend (BMFSFJ) (Hrsg.) (2011): Neue Wege - Gleiche Chancen. Gleichstellung von Frauen und Männern im Lebenslauf. Erster Gleichstellungsbericht. Berlin: BMFSFJ 2011.

Elder, Glen H. Jr. (1974): Children of the Great Depression. Social Change in Life Experience. Chicago: University of Chicago.

Friebel, Harald (2007): Soziale Sortierungen durchbrechen. Bildung und Weiterbildung im Lebenszusammenhang. In: Weiterbildung 18, 5, S. 2227.

Friebel, Harald (2008a): Die Kinder der Bildungsexpansion und das „Lebenslange Lernen". Augsburg: Ziel-Verlag.

Friebel, Harald (2008b): The Children of the Educational Expansion Era in Germany. In: British Journal of Sociology of Education 29, 5, S. 479492.

Friebel, Harald (2010): Weiterbildungsmarkt. In: Arnold. R./Nolda, S./ Nuissl, E. (Hrsg.):Wörterbuch Erwachsenenbildung. Bad Heilbrunn: Klinkhardt, S. 303-304.

Friebel, Harald et al. (2000): Bildungsbeteiligung: Chancen und Risiken. Eine Längsschnittstudie über Bildungs- und Weiterbildungskarieren in der „Moderne“. Opladen: Leske + Budrich.

Gerhard, Uta (1995). Typenbildung. In: Flick, U./Kardorff, E. v./Keupp, H./ Rosenstiel, L. v./Wolff, S. (Hrsg.): Handbuch qualitative Sozialfor- 
schung. Grundlagen, Konzepte, Methoden und Anwendungen. Zweite Auflage. Weinheim: Beltz, S. 432-434.

Glaser, Barney G./Strauss, Anselm L. (1967): The Discovery of Grounded Theory. Strategies for Qualitative Research. Chicago: Aldine.

Hartmann, Josef/Kuwan, Helmut (2011): Determinanten der Weiterbildungsbeteiligung: multivariate Analysen. In: Bilger, F./Rosenbladt, B. v. (Hrsg.): Weiterbildungsbeteiligung 2010. Trends und Analysen auf Basis des deutschen AES. Bielefeld: W. Bertelsmann Verlag, S. 70-79.

Haunberger, Sigrid (2011): Teilnahmeverweigerung in Panelstudien. Wiesbaden: VS Verlag für Sozialwissenschaften.

Helfferich, Cornelia (2012): Einleitung: Von roten Heringen, Gräben und Brücken. Versuche einer Kartierung von Agnecy-Konzepten. In: Bethmann, S. et al. (Hrsg.): Agency. Die Analyse von Handlungsfähigkeit und Handlungsmacht in qualitativer Sozialforschung und Gesellschaftstheorie. Weinheim, Basel: Beltz Juventa, S. 9-39.

Hoffmann, Heiko (2012): Relation, Identität und Agency. In: Bethmann et al. (Hrsg.): Agency. Die Analyse von Handlungsfähigkeit und Handlungsmacht in qualitativer Sozialforschung und Gesellschaftstheorie. Weinheim, Basel: Beltz Juventa, S. 154-181.

Keller, Bernd/Schulz, Susanne/Seifert, Hartmut (2012): Entwicklung und Strukturmerkmale der atypisch Beschäftigten in Deutschland bis 2010. WSI-Diskussionspapier Nr. 182. www.boeckler.de/pdf/p_wsi_182.pdf. [Zugriff: 21. Februar 2014].

Kluge, Susanne (1999): Empirisch begründete Typenbildung. Zur Konstruktion von Typen und Typologien in der qualitativen Sozialforschung. Opladen: Leske + Budrich.

Kluge, Susanne/Kelle, Udo (2010): Vom Einzelfall zum Typus. Zweite, überarbeitete Auflage. Wiesbaden: VS Verlag für Sozialwissenschaften.

Krüger, Helga (1995): Prozessuale Ungleichheit: Geschlecht und Institutionenverknüpfungen im Lebenslauf. In: Berger, P. A./Sopp, P. (Hrsg.): Sozialstruktur und Lebenslauf. Opladen: Leske + Budrich, S. 133-153.

Krüger, Helga (2002): Gesellschaftsanalyse: der Institutionenansatz in der Geschlechterforschung. In: Knapp. G. A./Wetterer, A. (Hrsg.): Soziale Verortung der Geschlechter. Münster: Verlag Westfälisches Dampfboot, S. 63-90.

Leven, Ingo/Bilger, Frauke/Strauß, Alexandra/Hartmann, Josef (2013): Weiterbildungstrends in verschiedenen Bevölkerungsgruppen. In: Bilger, F./Gnahs, D./Hartmann, J./Kuper, H. (Hrsg.): Weiterbildungsverhalten in Deutschland, Resultate des Adult Education Survey 2012, Bielefeld: W. Bertelsmann Verlag, S. 60-94.

Mayer, Karl-Ulrich/Blossfeld Hans-Peter (1990): Die gesellschaftliche Konstruktion sozialer Ungleichheit im Lebensverlauf. In: Berger, P. A./Hra- 
dil, S. (Hrsg.): Lebenslagen, Lebensläufe, Lebensstile. Soziale Welt, Sonderband 7. Göttingen: Otto Schwartz, S. 153-188.

Nader, Laima (2007): Berufliche Weiterbildung und Geschlechtergerechtigkeit. In: Report 30, 3, S. 29-38.

Projektgruppe Das Sozio-ökonomische Panel (DIW) (1998): Diskussionspapier Nr. 163. Funktion und Design einer Ergänzungsstichprobe für das sozioökonomische Panel (SOEP). Berlin: DIW. www.diw.de/documents/ publikationen/73/diw_01.c.38539.de/dp162.pdf. [Zugriff: 21. Februar 2014].

Rosenbladt, Bernhard von/Bilger, Frauke (Hrsg.) (2011): Weiterbildungsbeteiligung 2010. Bielefeld: W. Bertelsmann Verlag.

Schütze, Fritz (1981): Prozessstrukturen des Lebenslaufs. In: Matthes, J./ Pfeifenberg, A./Stosberg, M. (Hrsg.): Biografie in handlungswissenschaftlicher Perspektive. Wien: Verlag der Nürnberger Forschungsvereinigung, S. 67-156.

Strauss, Anselm/Corbin, Juliet (1990): Basics of Qualitative Research. London: Sage.

Stürzer, Monika (2005): Bildung, Ausbildung und Weiterbildung. In: Corneließen, W. (Hrsg.): Gender-Datenreport. 1. Datenreport zur Gleichstellung von Frauen und Männern in der Bundesrepublik Deutschland. München, S. 21-98. www.bmfsfj.de/doku/Publikationen/genderreport/01-Re daktion/PDF-Anlagen/gesamtdokument,property=pdf,bereich=genderrep ort,sprache $=$ de,rwb=true.pdf [Zugriff: 21 . Februar 2014].

Weber, Max (1904/1988): Die Objektivität sozialwissenschaftlicher und sozialpolitischer Erkenntnis. In: Weber, M. (Hrsg.): Gesammelte Aufsätze zur Wissenschaftslehre. Tübingen: Mohr Siebeck, S. 146-214.

West, Candace/Zimmermann, Don H. (1987): Doing Gender. In: Gender and Society 1, 2, S. 125-151.

Wittpoth, Jürgen (2009): Beteiligungsregulation in der Weiterbildung. In: Tippelt, R./von Hippel, A. (Hrsg.): Handbuch Erwachsenenbildung/Weiterbildung. Wiesbaden: VS Verlag für Sozialwissenschaften, S. 791-788. 